\title{
THE RESULTS OF THE STUDY OF THE EPIDEMIOLOGICAL STATUS AND SPREAD OF DERMACENTOR RETICULATUS TICKS IN UKRAINE OVER THE LAST 10 YEARS
}

DOI: $10.36740 /$ WLek202108130

\author{
Larysa Ya. Fedoniuk', Stepan S. Podobivskiy ${ }^{1}$, Iryna B. Pryvrotska' ${ }^{1}$ Olena A. Miklashevska ${ }^{2}$, Olga M. Marchuk ${ }^{1}$ \\ 'I. HORBACHEVSKY TERNOPIL NATIONAL MEDICAL UNIVERSITY, TERNOPIL, UKRAINE \\ 2TERNOPIL NON-COMMERCIAL ENTERPRISE "TERNOPIL COMMUNAL CITY HOSPITAL №2", TERNOPIL, UKRAINE
}

\begin{abstract}
The aim: To analyze the study of the $D$. reticulatus ticks epidemiology and to carry out their own examinations of ticks for their infection with pathogens of infectious diseases. Materials and methods: Identification of ticks was performed by an optoelectronic SEO system - IMAGLAB. Detection of pathogens in the studied ticks was carried out in research laboratory of $\mathrm{I}$. Horbachevsky TNMU by polymerase chain reaction (PCR) in real time using the amplifier "RotorGene - 6000 ".

Results: The review of scientific publications concerning an epidemiological condition of D. reticulatus ticks in particular countries of Europe and in Ukraine is carried out. According to the PCR results, 5 cases out of 21 samples of Borelia burgdorferi s.l. and Anaplasma phagocytophilum was detected. Researchers and students of I. Horbachevsky TNMU during 2017-2019 conducted field meetings in 74 locations from 10 regions of Ukraine: Lviv, Ivano-Frankivsk, Zakarpattia, Ternopil, Volyn, Rivne, Zhytomyr, Chernihiv, Khmelnytsky, Vinnytsia. In 2000-2018, researchers at the Department of Acarology of the I.I. Schmalhausen Institute of Zoology of the National Academy of Sciences of Ukraine the distribution of D. reticulatus in 311 locations in 79 settlements of its eastern and southern regions and the Autonomous Republic of Crimea revealed.

Conclusions: Medical geographic information system allows to create electronic cartographic models for scientific research and practical use for systematic monitoring, accounting and control of the medical and geographical situation of the study area on the prevalence of ticks and morbidity.
\end{abstract}

KEY WORDS: Ixodes ticks, Dermacentor reticulatus, distribution, epidemiology, Ukraine

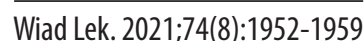

\section{INTRODUCTION}

Scientific fundamental research of Ixodes ticks in Ukraine began in the 60s of the twentieth century. In recent years, there is infectious diseases expansion associated with tick-transmitted pathogens, carriers, vectors of which are Ixodes tiks in the United States, and Europe including Ukraine is observed. For instance, tick-borne human grunocytic anaplasmosis (HGA) was first described in the United States in 1990 in Michigan, and between 1994 and 2004, 2,900 cases were reported [1]. Nowadays, Ixodes ticks in Ukraine are one of the numerous components of natural biocenoses and urbocenoses. Dermacentor reticulatus is one of the few species of Ixodes ticks that attack not only animals but also humans.

Although, there are still many unknown facts about the spreading of $D$. reticulatus in Ukraine and, in particular, its western regions [2]. Reports on the distribution of this specie, provided by a number of Ukrainian authors, is somewhat outdated.

Thus, the study of the prevalence of ticks of the genus Dermacentor reticulatus in different geographical areas, their ecological features, and biological patterns, epidemiological and medical significance remains a relevant topic for research. Based on the fact that this species of Ixodes ticks are an important vectors of the animals and human's infectious diseases pathogens transmission, the discovery of new places of its spread allows us to predict the spread of new infections in tick-occupied regions. That is why, the study of the epidemiological condition of ticks is an important aspect, which, in addition with the geographical distribution, allows us to predict the epidemiological status of different regions for tick borne infections.

\section{THE AIM}

To analyze the study of the $D$. reticulatus ticks epidemiology and to carry out their own examinations of ticks for their infection with pathogens of infectious diseases. Summarize the data of field collections of ticks of this species, conducted by scientists of I. Horbachevsky Ternopil National Medical University during 2017-2020 and researchers of the Department of Acarology of the I.I. Schmalhausen Institute of Zoology of the National Academy of Sciences of Ukraine in 2000-2018 on its distribution in the western, eastern and southern regions of Ukraine and in the Autonomous Republic of Crimea. To reveal the results of research of some aspects of biology of this species. 


\section{MATERIALS AND METHODS}

The collection of ticks was carried out by authors and volunteers - students of the Medical University at their place of residence, as well as researchers of the Department of Acarology of the I.I. Schmalhausen Institute of Zoology of the National Academy of Sciences of Ukraine. It was mainly used to manually collect ticks from animals and remove ticks from grass using a "flag" method. Ticks were identified using the optoelectronic SEO system - IMAGLAB. To determine the ticks, the tables for determination given in the monographs of I.A. Akimov and E.M. Yemchuk were used.

Detection of pathogens in the studied ticks was carried out on the basis of the sector of experimental and clinical research of the interdepartmental training and research laboratory of I. Horbachevsky TNMU by the method of polymerase chain reaction (PCR) in real time using the amplifier "RotorGene-6000". To do this, prepare a suspension of each mite separately. VectorBest detection kit was used as a transport medium with cryopreservatives and as a solution for ticks preparation. In particular, "RealBest DNA Borrelia burgdorferi senso lato", "RealBest DNA Borrelia Miyamotoi", "RealBest DNA Anaplasma phagocytophilum / Ehrlichia muris / Ehrlichia haffeensis", "RealBest DNA in Babesia species", "RealBest RNA in Babesia species" set. A kit from the same company was used for DNA / RNA extraction.

By conducting a polymerase chain reaction was detected DNA of B. burgdorferi senso lato, B. Myamotoi, Anaplasma phagocytofilum / Erlichia muris / E. chaafeensis, Babesia sp. and tick-borne encephalitis virus (TBEV) RNA.

\section{RESULTS AND DISCUSSION}

Scientists in many countries in Europe and North America pay special attention to the study of the possibility of transmission of Ixodes ticks, including D. reticulatus, pathogens. So, Ewa J. Mierzejewska et al [3] indicate that in the eastern (5.42\%) and northeastern (2.32\%) regions of ticks of this species are carriers of Babesia canis. In the same regions, $52.03 \%$ and $41.75 \%$ of ticks, respectively, are carriers of Rickettsia raoulti. There are some data on the infection of D. reticulatus with spirochetes Borelia burgdorferi sl. Thus, in the western regions of the Republic of Poland, only $0.009 \%$ of ticks are carriers of these bacteria, and in the territory of the Lubelskie Voivodeship - 0.6\% [4]. In France, this figure is 1.5\% [5], and in the Republic of Belarus - 2,7\% [6]. D. reticulatus has been shown to transmit tick-borne encephalitis viruses (TBEV). Thus, in particular, in eastern Poland, the level of infection of ticks of this species with the corresponding virus was more than $10.8 \%$ [7]. Numerous studies indicate the ability of $D$. reticulatus to tolerate Anaplasma phagocytophilum in many European countries, including Serbia [8].

Nowadays Ixodes ticks are one of the numerous components of natural biocenoses and urbocenoses in Ukraine, especially in its western regions. They can be observed in large quantities not only in sparse forests, on the edges, meadows and steppe areas, but also within various settlements, including large cities. Nowadays Ixodes ticks can be detected in parks, squares, recreational areas and even in small greenery along the streets or near residential buildings. This certain larva and nymph's ticks species spread can be explained by the presence of murine rodents and birds hosts, in which they are feed on; cats and dogs are excellent feeders for the adult stages of ticks $[2,9]$.

One of the first data on the habitats of $D$. reticulatus within Ukraine were presented in the monograph EM Yemchuk [10]. According to these data, this tick specie is found in 10 Ukraine regions (Lviv, Ternopil, Volyn, Rivne, Khmelnytsky, Vinnytsia, Kyiv, Sumy, Zhytomyr, Chernihiv), occupying mainly forest habitats.

The issue of distribution and biology of Ixodes ticks, including ticks of the genus Dermacentor in the Carpathians and Transcarpathian region was dealt with by II Turyanin [11], in which, he indicates the findings of ticks of this species in the Transcarpathian, Ivano-Frankivsk, Lviv and Chernivtsi regions.

There are some scientific sources on the distribution of $D$. reticulatus in urban areas. Thus, the monograph and scientific articles of I.A. Akimov and I.V. Nebogatkin provide data on the distribution of that species in the biotopes of Kyiv [12,13]. According to the results of research carried out by scientists in 1988, 1991-1992, 2008-2009, the southern areas of $D$. reticulatus were established, including in the Crimea.

Regularities of attraction of this species to settlements, including cities, are revealed. G.V. Kolonin covers the study of prevalence of ticks of the genus Dermacentor in the Eastern and Western Europe in detail in the monograph in 1984 [14].

I.G. Uspenska is revealed the finding of D. reticulatus in the Moldavian codras (mountainous terrain) [15].

The most complete research of ticks prevalence in the Republic of Belarus, including the biology and epidemiology of pasture ticks, is discovered in the monograph E.I. Bychkova, I.A. Fedorova, M.M. Yakovich [16].

There are numerous studies of pasture ticks in the Poland. Thus, Waldemar Biadun studied this specie within Lublin [17], Ewa J. Mierzejewska and her colleagues [3] studied D. reticulatus in its central regions, Anna Paziewska and colleagues [18] studied the prevalence of this specie in the northern regions of Poland, Zygner W. [19] and other scientists study the infection by Babesia species of $D$. reticulatus ticks in central Poland.

Lydia Chitimia-Dobler studied the distribution of $D$. reticulatus in Romania [20]. The biotope distribution of this specie was studied by Eva Bullová, Martin Lukáň, Michal Stanko, Branislav Petko in Slovakia [21]. Scientists Földvári G. have made an important contribution to the study of morphological features, biology, epidemiological significance and geographical distribution of D. reticulatus in Hungary [22, 23].

At the sector of experimental and clinical research of the interdepartmental educational-research laboratory of I. Horbachevsky TNMU, real-time PCR was used to study ticks from 21 locations using the RotorGene-6000 amplifier. In 5 cases, the fact of infection of ticks with pathogens: 3 - Borelia burgdorferi s.1, 1 - Anaplasma phagocytophilum and 1 - mixed infection of B. burgdorferi with A. phagocytophilum was detected. 
Table I. Distribution and biology of ticks of the species Dermacentor reticulatus (according to research by the laboratory of TNMU)

\begin{tabular}{|c|c|c|c|c|}
\hline $\begin{array}{l}\text { Date of tick } \\
\text { detection }\end{array}$ & $\begin{array}{l}\text { Meeting place: region, district, settlement, } \\
\text { geographical coordinates of the area }\end{array}$ & $\begin{array}{l}\text { The host } \\
\text { from which } \\
\text { the tick was } \\
\text { extracted }\end{array}$ & $\begin{array}{c}\text { Number } \\
\text { of ticks } \\
\text { on one } \\
\text { host }\end{array}$ & $\begin{array}{c}\text { The } \\
\text { pathogen } \\
\text { detected by } \\
\text { PCR }\end{array}$ \\
\hline \multicolumn{5}{|c|}{ Ternopil region } \\
\hline 5.05 .2017 & 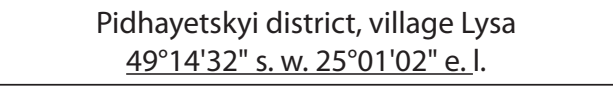 & dog & $\begin{array}{c}1 \text { male, } \\
2 \text { females }\end{array}$ & $* *$ \\
\hline 5.05 .2017 & $\begin{array}{l}\text { Ternopil district, c. Ivachiv } \\
49^{\circ} 38^{\prime} 45^{\prime \prime} \text { n. w. } 25^{\circ} 32^{\prime} 54^{\prime \prime} \text { e. I. } \\
\end{array}$ & cat & $\begin{array}{l}1 \text { male, } \\
1 \text { female }\end{array}$ & * \\
\hline 10.09.2017. & $\begin{array}{l}\text { Kremenets district, w. Roztoky } \\
49^{\circ} 55^{\prime} 02^{\prime \prime} \text { n. w. } 25^{\circ} 31^{\prime} 24^{\prime \prime} \text { e. I. }\end{array}$ & cow & $\begin{array}{l}4 \text { males, } \\
4 \text { females }\end{array}$ & $* *$ \\
\hline 10.09 .2017 & $\begin{array}{l}\text { Zbarazh district, c. Zbarazh } \\
49^{\circ} 40^{\prime} 17^{\prime \prime} \text { n. w. } 25^{\circ} 46^{\prime} 15^{\prime \prime} \text { e. I. } \\
\end{array}$ & man & 1 male & * \\
\hline 11.09.2017 & \multirow{10}{*}{$\begin{array}{c}\text { c. Ternopil } \\
49^{\circ} 34^{\prime} \text { n. w. } 25^{\circ} 36^{\prime} \text { e. I. }\end{array}$} & man & 1 female & * \\
\hline 06.10 .2017 & & man & 1 male & * \\
\hline 04.05.2018 & & man & 1 female & * \\
\hline 04.06.2018 & & man & 1 female & * \\
\hline 11. 11.2018 & & dog & 1 male, & * \\
\hline & & & 2 females & * \\
\hline 16.10 .2018 & & man & 1 female & $*$ \\
\hline 02.04.2019 & & man & 1 male & $\mathrm{Bb}$ \\
\hline 14.06.2019 & & man & 1 female & $A$ \\
\hline 02.10 .2019 & & man & 1 female & $*$ \\
\hline 6.10 .2017 & $\begin{array}{c}\text { Ternopil district, v. Velykyy Hlybochok } \\
49^{\circ} 37^{\prime} 16^{\prime \prime} \text { n. w. } 25^{\circ} 31^{\prime} 51^{\prime \prime} \text { e.l. } \\
\end{array}$ & man & 1 male & * \\
\hline 10.10.2017 & $\begin{array}{l}\text { Lanovets district, c. Lanivtsi } \\
49^{\circ} 52^{\prime} 00^{\prime \prime} \text { n. w. } 26^{\circ} 05^{\prime} 14^{\prime \prime} \text { e.l. }\end{array}$ & dog & 1 female & $* *$ \\
\hline 10.10 .2017 & $\begin{array}{c}\text { Bereazhanskyy district, v. Zhukiv } \\
49^{\circ} 30^{\prime} 57^{\prime \prime} \text { n. w. } 24^{\circ} 56^{\prime} 27^{\prime \prime} \text { e.l. }\end{array}$ & man & 1 female & $\mathrm{Bb}$ \\
\hline 18.10.2017 & $\begin{array}{l}\text { Ternopil district, v. Petryky } \\
49^{\circ} 31^{\prime} 40^{\prime \prime} \text { n. w. } 25^{\circ} 34^{\prime} 38^{\prime \prime} \text { e.l. } \\
\end{array}$ & man & 1 female & * \\
\hline 2.11 .2017 & $\begin{array}{l}\text { Lanovets district, v. Plyska } \\
49^{\circ} 44^{\prime} 39^{\prime \prime} \text { n. w. } 26^{\circ} 05^{\prime} 40^{\prime \prime} \text { e.l. }\end{array}$ & dog & $\begin{array}{l}2 \text { males, } \\
2 \text { females }\end{array}$ & $* *$ \\
\hline 5.11 .2017 & $\begin{array}{l}\text { Lanovets district, v. Molotkiv } \\
\underline{49^{\circ} 49^{\prime} 29^{\prime \prime} \text { n. w. } 26^{\circ} 11^{\prime} 17^{\prime \prime} \text { e.l. }} \\
\end{array}$ & cat & $\begin{array}{l}1 \text { female } \\
1 \text { male }\end{array}$ & $* *$ \\
\hline 07.11 .2017 & $\begin{array}{c}\text { Ternopil district, v. Bila } \\
49^{\circ} 35^{\prime} 03^{\prime \prime} \text { n. w. } 25^{\circ} 34^{\prime} 41^{\prime \prime} \text { e.l. }\end{array}$ & man & 1 female & * \\
\hline 6.10 .2018 & $\begin{array}{l}\text { Zboriv district, c. Pidberiztsi } \\
49^{\circ} 51^{\prime} 26^{\prime \prime} \text { n. w. } 25^{\circ} 20^{\prime} 49^{\prime \prime} \text { e.l. }\end{array}$ & dog & 3 females & $* *$ \\
\hline 14.04.2019 & $\begin{array}{l}\text { Ternopil district. Hayi Shevchenkivski } \\
\underline{49^{\circ} 36^{\prime} 05^{\prime \prime} \text { n. w. } 25^{\circ} 37^{\prime} 48^{\prime \prime} \text { e.l. }}\end{array}$ & man & 1 female & * \\
\hline 23.04.2019 & $\begin{array}{l}\text { Ternopil district, v. Lozova } \\
49^{\circ} 36^{\prime} 39^{\prime \prime} \text { n. w. } 25^{\circ} 40^{\prime} 13^{\prime \prime} \text { e.l. } \\
\end{array}$ & dog & $\begin{array}{c}1 \text { male, } \\
2 \text { females }\end{array}$ & $* *$ \\
\hline 03.05.2019 & $\begin{array}{c}\text { c. Shumsk } \\
50^{\circ} 06^{\prime} 51^{\prime \prime} \text { n. w. } 26^{\circ} 06^{\prime} 52^{\prime \prime} \text { e.l. } \\
\end{array}$ & dog & $\begin{array}{l}5 \text { males, } \\
1 \text { female }\end{array}$ & $* *$ \\
\hline 30.09 .2019 & $\begin{array}{c}\text { Kremenets district, v. Losyatyn } \\
49^{\circ} 58^{\prime} 16^{\prime \prime} \text { n. w. } 25^{\circ} 29^{\prime} 23^{\prime \prime} \text { e.l. }\end{array}$ & dog & 6 female & $* *$ \\
\hline 29.10.2019 & $\begin{array}{c}\text { Pidvolochysk district, v. Skoryky } \\
49^{\circ} 35^{\prime} 49^{\prime \prime} \text { n. w. } 26^{\circ} 08^{\prime} 33^{\prime \prime} \text { e.l. }\end{array}$ & cow & $\begin{array}{l}2 \text { males, } \\
7 \text { females }\end{array}$ & $* *$ \\
\hline 18.11.2019 & $\begin{array}{l}\text { Chortkiv district, v. Palashivka } \\
48^{\circ} 58^{\prime} 37^{\prime \prime} \text { n. w. } 25^{\circ} 34^{\prime} 13^{\prime \prime} \text { e.l. }\end{array}$ & dog & $\begin{array}{l}2 \text { males, } \\
5 \text { females }\end{array}$ & $* *$ \\
\hline 2.12 .2019 & $\begin{array}{c}\text { Borshchiv district, c. Borshchiv } \\
48^{\circ} 48^{\prime} 05^{\prime \prime} \text { n. w. } 26^{\circ} 02^{\prime} 32^{\prime \prime} \text { e.l. }\end{array}$ & cat & $\begin{array}{c}1 \text { male, } \\
2 \text { females }\end{array}$ & $* *$ \\
\hline
\end{tabular}


THE RESULTS OF THE STUDY OF THE EPIDEMIOLOGICAL STATUS AND SPREAD OF DERMACENTOR RETICULATUS...

\section{Lviv region}

\begin{tabular}{|c|c|c|c|c|}
\hline 29.05.2017 & $\begin{array}{c}\text { Busk district, m. Ostrivchyk-Pylnyy } \\
49^{\circ} 53^{\prime} 41^{\prime \prime} \text { n. w. } 24^{\circ} 43^{\prime} 33^{\prime \prime} \text { e.l. }\end{array}$ & horse & $\begin{array}{l}4 \text { males, } \\
8 \text { female }\end{array}$ & $\mathrm{Bb}$ \\
\hline 10.10.2017 & $\begin{array}{c}\text { Zolochiv district, c. Pidhorodnye } \\
49^{\circ} 47^{\prime} 34^{\prime \prime} \text { n. w. } 24^{\circ} 55^{\prime} 38^{\prime \prime} \text { e.l. }\end{array}$ & dog & $\begin{array}{l}7 \text { males, } \\
3 \text { females }\end{array}$ & $* *$ \\
\hline 23.04.2019 & $\begin{array}{c}\text { c. Socal } \\
50^{\circ} 29^{\prime} \text { n. w. } 24^{\circ} 17^{\prime} \text { e.l. } \\
\end{array}$ & $\operatorname{dog}$ & $\begin{array}{l}2 \text { males, } \\
4 \text { females }\end{array}$ & $* *$ \\
\hline 3.05 .2019 & $\begin{array}{l}\text { Yavoriv district, v. Losyno } \\
49^{\circ} 56^{\prime} 52^{\prime \prime} \text { n. w. } 23^{\circ} 48^{\prime} 53^{\prime \prime} \text { e.l. } \\
\end{array}$ & cow & $\begin{array}{c}1 \text { male, } \\
6 \text { female }\end{array}$ & $* *$ \\
\hline 15.09.2019 & $\begin{array}{c}\text { Lviv } \\
49^{\circ} 50^{\prime} 30^{\prime \prime} \text { n. w. } 24^{\circ} 01^{\prime} 53^{\prime \prime} \text { e.l. } \\
\end{array}$ & $\operatorname{dog}$ & $\begin{array}{c}2 \text { males, } \\
2 \text { females }\end{array}$ & $* *$ \\
\hline 10.10.2019 & $\begin{array}{l}\text { Busky district, v. Bezbrody } \\
49^{\circ} 55^{\prime} 06^{\prime \prime} \text { n. w.. } 24^{\circ} 33^{\prime} 10^{\prime \prime} \text { e.l. } \\
\end{array}$ & cow & $\begin{array}{l}8 \text { males, } \\
6 \text { female }\end{array}$ & $* *$ \\
\hline 15.10 .2019 & $\begin{array}{c}\text { Sokal district, v. Silets } \\
50^{\circ} 17^{\prime} 57^{\prime \prime} \text { n. w.241' } 11^{\prime \prime} 53^{\prime \prime} \text { e.l. }\end{array}$ & cow & 6 female & $* *$ \\
\hline 1.11 .2019 & 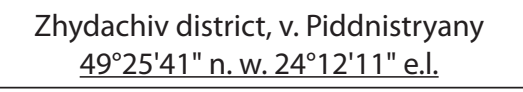 & $\operatorname{dog}$ & 2 females & $* *$ \\
\hline \multicolumn{5}{|c|}{ Ivano-Frankivsk region } \\
\hline 01.04 .2017 & $\begin{array}{l}\text { Kolomyia district, v. Runguri } \\
48^{\circ} 28^{\prime} 41^{\prime \prime} \text { n. w. } 24^{\circ} 51^{\prime} 38^{\prime \prime} \text { e.l. }\end{array}$ & $\operatorname{dog}$ & 4 males & $\mathrm{A}, \mathrm{Bb}$ \\
\hline 5.05 .2017 & $\begin{array}{l}\text { Yaremche district, Yaremche } \\
48^{\circ} 27^{\prime} 37^{\prime \prime} \text { n. w. } 24^{\circ} 33^{\prime} 31^{\prime \prime} \text { e.l. }\end{array}$ & $\operatorname{dog}$ & 1 female & * \\
\hline 10.05.2017 & $\begin{array}{c}\text { Kalush district, Kalush } \\
49^{\circ} 02^{\prime} 39^{\prime \prime} \text { n. w. } 24^{\circ} 21^{\prime} 35^{\prime \prime} \text { e.l. } \\
\end{array}$ & $\operatorname{dog}$ & 2 females & $* *$ \\
\hline \multicolumn{5}{|c|}{ Transcarpathian region } \\
\hline 14.06.2017 & $\begin{array}{c}\text { Mukachevo } \\
48^{\circ} 26^{\prime} 29^{\prime \prime} \text { n. w. } 22^{\circ} 42^{\prime} 49^{\prime \prime} \text { e.l. } \\
\end{array}$ & dog & $\begin{array}{l}1 \text { male, } \\
1 \text { female }\end{array}$ & $* *$ \\
\hline 03.05.2019 & $\begin{array}{c}\text { Khust district, v. Kireshi } \\
48^{\circ} 11^{\prime} 20^{\prime \prime} \text { n. w. } 23^{\circ} 21^{\prime} 09^{\prime \prime} \text { e.l. } \\
\end{array}$ & $\operatorname{dog}$ & $\begin{array}{l}1 \text { male, } \\
3 \text { females }\end{array}$ & $* *$ \\
\hline \multicolumn{5}{|c|}{ Volyn region } \\
\hline 29.05.2017 & $\begin{array}{l}\text { Gorokhiv district, v. Yarivka } \\
\underline{50^{\circ} 32^{\prime} 54^{\prime \prime} \text { n.w. } 24^{\circ} 54^{\prime} 04^{\prime \prime} \text { e.l. }}\end{array}$ & $\operatorname{dog}$ & $\begin{array}{l}7 \text { males, } \\
5 \text { female }\end{array}$ & * \\
\hline 15.09.2017 & $\begin{array}{l}\text { Lyubeshiv district, v. Prochody } \\
51^{\circ} 47^{\prime} 10^{\prime \prime} \text { n.w. } 25^{\circ} 28^{\prime} 11^{\prime \prime} \text { e.I. }\end{array}$ & dog & $\begin{array}{l}6 \text { males, } \\
4 \text { females }\end{array}$ & $* *$ \\
\hline 19.09.2017 & $\begin{array}{l}\text { Lyuboml district, v. Gushcha } \\
\underline{51^{\circ} 16^{\prime} 44^{\prime \prime} \text { n.w. } 23^{\circ} 45^{\prime} 06^{\prime \prime} \text { e.l. }} \\
\end{array}$ & cow & 6 males & $* *$ \\
\hline 8.10 .2017 & $\begin{array}{l}\text { Kivertsiv district, v.Pokaschiv } \\
\underline{50^{\circ} 44^{\prime} 51^{\prime \prime} \text { n.w. } 25^{\circ} 41^{\prime} 23^{\prime \prime} \text { e.I. }}\end{array}$ & cow & $\begin{array}{l}4 \text { males, } \\
21 \text { female }\end{array}$ & $* *$ \\
\hline 10.11 .2017 & $\begin{array}{c}\text { Gorokhiv district, v.Lobachivka } \\
50^{\circ} 25^{\prime} 15^{\prime \prime} \text { n.w. } 24^{\circ} 58^{\prime} 05^{\prime \prime} \text { e.l. }\end{array}$ & cow & $\begin{array}{l}8 \text { males, } \\
8 \text { female }\end{array}$ & $* *$ \\
\hline 2.11 .2018 & 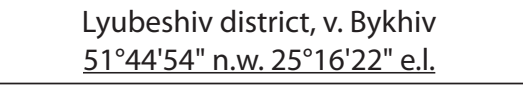 & $\operatorname{dog}$ & $\begin{array}{l}4 \text { males, } \\
5 \text { female }\end{array}$ & $* *$ \\
\hline 23.04. 2019 & $\begin{array}{l}\text { Lutsk district, v. Velykyy Omelyanik } \\
50^{\circ} 44^{\prime} 19^{\prime \prime} \text { n.w. } 25^{\circ} 16^{\prime} 04^{\prime \prime} \text { e.l. }\end{array}$ & $\operatorname{dog}$ & 1 male & $* *$ \\
\hline 15.10 .2019 & $\begin{array}{l}\text { Manevychi district, v. Kukly } \\
51^{\circ} 13^{\prime} 28^{\prime \prime} \text { n.w. } 25^{\circ} 38^{\prime} 53^{\prime \prime} \text { e.l. }\end{array}$ & cow & $\begin{array}{l}1 \text { male, } \\
6 \text { females }\end{array}$ & $* *$ \\
\hline 18.10.2019 & $\begin{array}{l}\text { Volodymyr-Volynskyi district, v. Rusniv } \\
\underline{50^{\circ} 44^{\prime} 53^{\prime \prime} \text { n.w. } 24^{\circ} 19^{\prime} 08^{\prime \prime} \text { e.l. }}\end{array}$ & cow & $\begin{array}{l}3 \text { males, } \\
2 \text { females }\end{array}$ & $* *$ \\
\hline \multicolumn{5}{|c|}{ Rivne region } \\
\hline 15.04 .2017 & $\begin{array}{c}\text { Goshchanskyi district, v. Bugryn } \\
\underline{50^{\circ} 32^{\prime} 16^{\prime \prime} \text { n.w. } 26^{\circ} 31 \text { '38" e.l. }}\end{array}$ & dog & $\begin{array}{l}4 \text { males, } \\
9 \text { female }\end{array}$ & $* *$ \\
\hline 28.09.2017 & $\begin{array}{l}\text { Ostroh district, village Mezhyrich } \\
50^{\circ} 18^{\prime} 04^{\prime \prime} \text { n.w. } 26^{\circ} 28^{\prime} 30^{\prime \prime} \text { e.l. }\end{array}$ & man & 1 female & $* *$ \\
\hline
\end{tabular}




\begin{tabular}{|c|c|c|c|c|}
\hline 12.10 .17 & 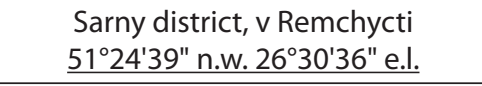 & $\operatorname{dog}$ & $\begin{array}{l}11 \text { males, } \\
5 \text { females }\end{array}$ & ** \\
\hline 23.10 .2017 & $\begin{array}{c}\text { Volodymyretskyi district, v. Svaryny } \\
51^{\circ} 16^{\prime} 43^{\prime \prime} \text { n.w. } 26^{\circ} 15^{\prime} 16^{\prime \prime} \text { e.I. }\end{array}$ & cow & $\begin{array}{l}3 \text { males, } \\
7 \text { females }\end{array}$ & ** \\
\hline 07.09 .2018 & Dubno $50^{\circ} 23^{\prime} 35^{\prime \prime}$ n.w. $25^{\circ} 44^{\prime} 06^{\prime \prime}$ e.l. & cow & 1 female & ** \\
\hline 08.09.2018 & $\begin{array}{l}\text { Mlyniv district, v. Vovnychy } \\
\underline{50^{\circ} 30^{\prime} 56^{\prime \prime} \text { n.w. } 25^{\circ} 24^{\prime} 19^{\prime \prime} \text { e.l. }}\end{array}$ & cow & $\begin{array}{l}4 \text { males, } \\
3 \text { females }\end{array}$ & ** \\
\hline 20.09.2019 & $\begin{array}{c}\text { Rivne } \\
\text { 50³7'11" n.w. } 26^{\circ} 15^{\prime} 05^{\prime \prime} \text { e.l. }\end{array}$ & cat & $\begin{array}{l}1 \text { male, } \\
1 \text { female }\end{array}$ & ** \\
\hline 2.10 .2019 & $\begin{array}{l}\text { Kostopil district, v Penjkiv } \\
51^{\circ} 01^{\prime} 43^{\prime \prime} \text { n.w. } 26^{\circ} 27^{\prime} 24^{\prime \prime} \text { e.l. }\end{array}$ & cow & $\begin{array}{l}6 \text { males, } \\
4 \text { females }\end{array}$ & ** \\
\hline 6.05 .2019 & 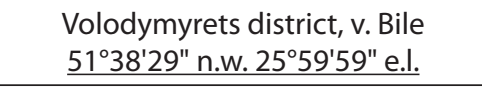 & $\operatorname{dog}$ & $\begin{array}{l}3 \text { males, } \\
7 \text { females }\end{array}$ & ** \\
\hline 8.05 .2019 & $\begin{array}{c}\text { Sarny district, Sarny } \\
51^{\circ} 19^{\prime} 37^{\prime \prime} \text { n.w. } 26^{\circ} 37^{\prime} 59^{\prime \prime} \text { e.l. } \\
\end{array}$ & horse & $\begin{array}{l}2 \text { males, } \\
8 \text { female }\end{array}$ & ** \\
\hline \multicolumn{5}{|c|}{ Zhytomyr region } \\
\hline 13.05 .2017 & $\begin{array}{c}\text { Korostyshiv } \\
50^{\circ} 19^{\prime} 07^{\prime \prime} \text { n.w. } 29^{\circ} 03^{\prime} 33^{\prime \prime} \text { e.l. }\end{array}$ & $\operatorname{dog}$ & 2 males & * \\
\hline 22.05 .2017 & $\begin{array}{c}\text { Lyubar district, v. Krasnovolitsa } \\
50^{\circ} 01^{\prime} 56^{\prime \prime} \text { n.w. } 28^{\circ} 00^{\prime} 44^{\prime \prime} \text { e.l. }\end{array}$ & dog & 6 females & ** \\
\hline 20.09.2018 & $\begin{array}{c}\text { Olevsky district, Olevsk } \\
51^{\circ} 13^{\prime} 40^{\prime \prime} \text { n.w. } 27^{\circ} 38^{\prime} 53^{\prime \prime} \text { e.l. } \\
\end{array}$ & $\operatorname{dog}$ & 2 females & ** \\
\hline \multicolumn{5}{|c|}{ Khmelnytsky region } \\
\hline 3.10 .2017 & $\begin{array}{c}\text { Khmelnytsky } \\
49^{\circ} 25^{\prime} 10^{\prime \prime} \text { n.w. } 26^{\circ} 58^{\prime} 46^{\prime \prime} \text { e.l. } \\
\end{array}$ & dog & $\begin{array}{c}1 \text { male, } \\
15 \text { females }\end{array}$ & ** \\
\hline 14.10.2017 & $\begin{array}{c}\text { Yemilchevskyi district, t.Yemilchene } \\
\underline{50^{\circ} 52^{\prime} 15^{\prime \prime} \text { n.w. } 27^{\circ} 48^{\prime} 26^{\prime \prime} \text { e.l. }}\end{array}$ & dog & $\begin{array}{c}5 \text { males, } \\
23 \text { femaless }\end{array}$ & ** \\
\hline 16.09.2017 & $\begin{array}{c}\text { Derazhnyansky district, t.Lozove } \\
49^{\circ} 17^{\prime} 35^{\prime \prime} \text { n.w. } 27^{\circ} 17^{\prime} 57^{\prime \prime} \text { e.l. }\end{array}$ & flag & $\begin{array}{l}7 \text { males, } \\
6 \text { females }\end{array}$ & ** \\
\hline 12.10 .2017 & $\begin{array}{l}\text { Belogorsky district, v. Sushivcti } \\
49^{\circ} 58^{\prime} 35^{\prime \prime} \text { n.w. } 26^{\circ} 21^{\prime} 02^{\prime \prime} \text { e.l. }\end{array}$ & cow & 7 females & ** \\
\hline 10.10.2018 & $\begin{array}{l}\text { Vinkovets district, Vinkivtsi } \\
49^{\circ} 01^{\prime} 59^{\prime \prime} \text { n.w. } 27^{\circ} 14^{\prime} 01^{\prime \prime} \text { e.l. } \\
\end{array}$ & dog & $\begin{array}{l}5 \text { males, } \\
4 \text { females }\end{array}$ & ** \\
\hline 25.10 .2018 & $\begin{array}{c}\text { Slavutych district, Slavuta } \\
50^{\circ} 18^{\prime} \text { ' n.w. } 26^{\circ} 52^{\prime} \text { e.l. }\end{array}$ & flag & $\begin{array}{l}1 \text { male, } \\
1 \text { female }\end{array}$ & ** \\
\hline 23.04 .2019 & 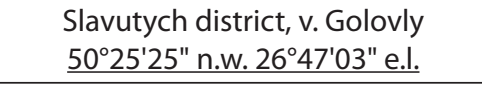 & horse & $\begin{array}{l}5 \text { males, } \\
6 \text { females }\end{array}$ & ** \\
\hline 26.04 .2019 & $\begin{array}{c}\text { Yarmolynets district, v. Golohvasti } \\
49^{\circ} 16^{\prime} 30^{\prime \prime} \text { n.w. } 26^{\circ} 57^{\prime} 46^{\prime \prime} \text { e.l. }\end{array}$ & dog & 6 female & ** \\
\hline \multicolumn{5}{|c|}{ Vinnytsia region } \\
\hline 10.05.2017 & $\begin{array}{c}\text { Barsky district, c. Luka Barska } \\
49^{\circ} 09^{\prime} 09^{\prime \prime} \text { n.w. } 27^{\circ} 47^{\prime} 54^{\prime \prime} \text { e.l. }\end{array}$ & cow & $\begin{array}{l}1 \text { male, } \\
8 \text { females }\end{array}$ & ** \\
\hline 02.05 .2019 & $\begin{array}{c}\text { c. Illintsi } \\
49^{\circ} 06^{\prime} \text { ' n.w. } 29^{\circ} 12^{\prime} \text { e.l. }\end{array}$ & dog & $\begin{array}{l}5 \text { males, } \\
8 \text { females }\end{array}$ & ** \\
\hline \multicolumn{5}{|c|}{ Chernihiv region } \\
\hline 06.09 .2018 & $\begin{array}{l}\text { Nizhyn district, v. Lypiv Rig } \\
51^{\circ} 04^{\prime} 41^{\prime \prime} \text { n.w. } 31^{\circ} 57^{\prime} 07^{\prime \prime} \text { e.l. }\end{array}$ & dog & $\begin{array}{l}1 \text { male, } \\
5 \text { females }\end{array}$ & ** \\
\hline
\end{tabular}

Note: A - Anaplasma phagocytophilum; Bb - Borrelia burgdorferi s.l.;

*- no pathogens were detected; ${ }^{* *}-P C R$ analysis was not performed.

An important task of the our research was not only to identify the species composition of ticks and their life forms, but also to study the biology and epidemiology of species that attack humans and animals and are able to transmit pathogens to them [24, 25]. During May 2017 - November 2019, the laboratory identified 447 specimens of ticks of the species $D$. reticulatus, including 287 females and 160 males, collected in 66 settlements from 10 regions of Ukraine. 


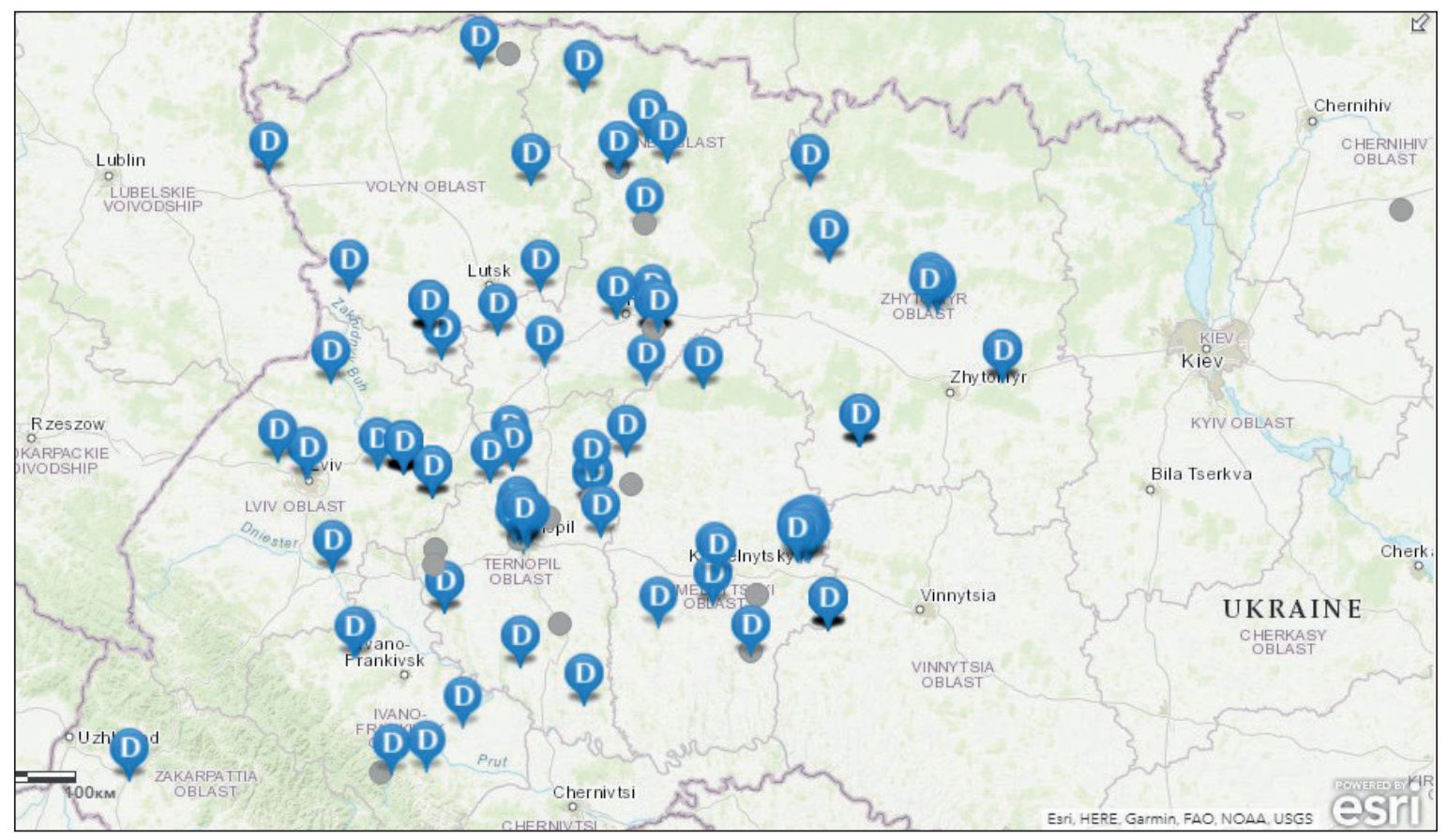

Fig. 1. Data on the spread of D. reticulatus ticks in Ukraine (according to the results of processing in the laboratory of TNMU)

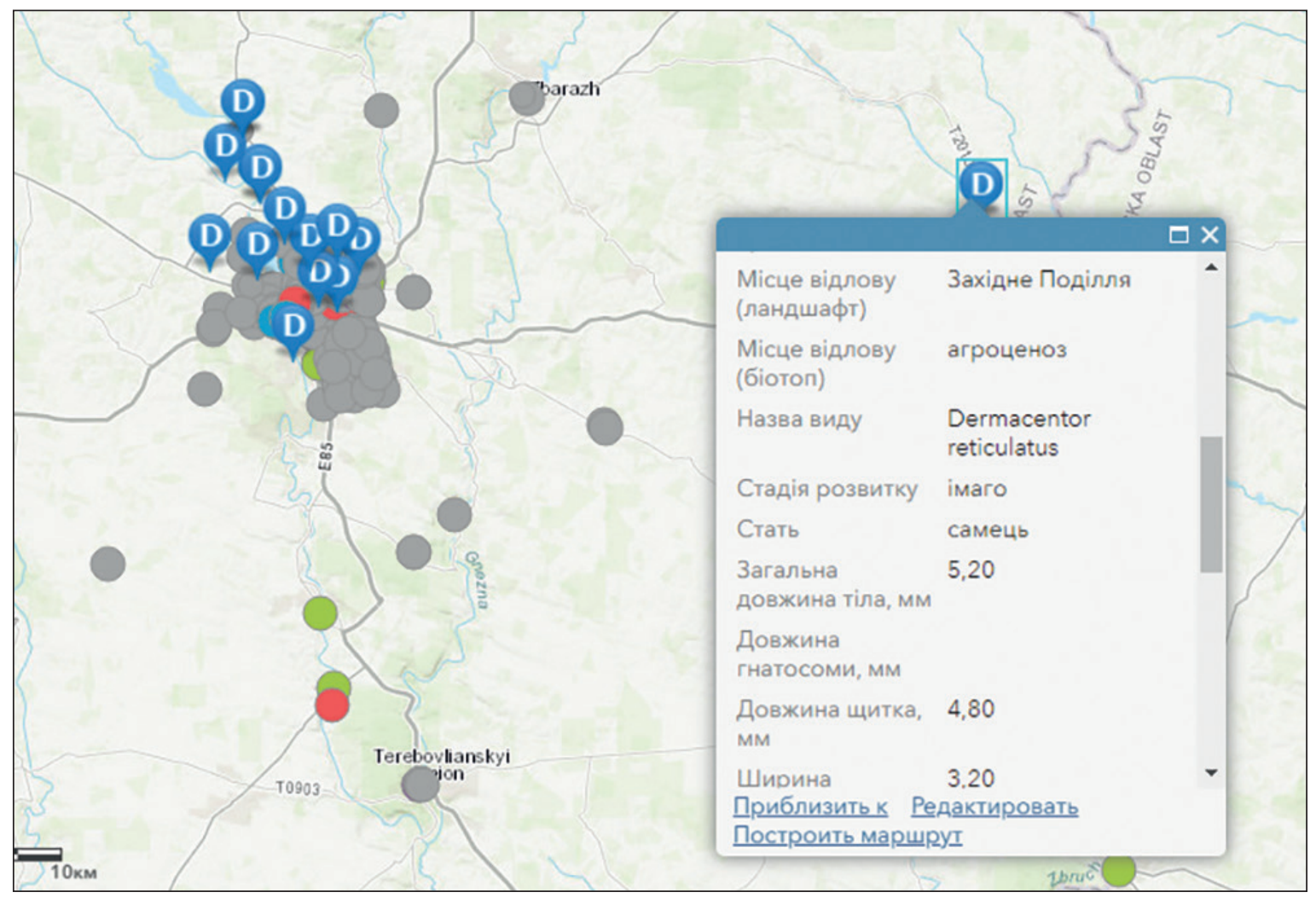

Fig. 2. Places of collection of ticks and registration of examined patients affected by ticks in the Ternopil region. Note: the examined patients are marked with circles (different colors indicate the types of employment of patients)

We recorded 74 cases ticks detection on animals, humans and in grassland, which is shown in table 1 . The main feeding hosts, on which adult ticks of both sexes were found, were 4 species of animals: dogs -34 cases, cows -16 , cats -4 , horses - 3, humans - 15 cases, in 2 cases ticks were caught on the "flag". Compared to other Ixodes ticks, in particular
Ixodes ricinus, $D$. reticulatus is much less likely to attack humans, but mostly attacks animals. It was found that $D$. reticulatus attacked humans only as adult males and females (the ratio between the sexes is almost proportional), while I. ricinus parasitizes humans at almost all stages: larvae, nymphs, adults. 
During 2000-2018, researchers of the Department of Acarology of the I.I. Schmalhausen Institute of Zoology of the National Academy of Sciences of Ukraine carried out field research to detect ticks of the species I. ricinus and D. reticulatus in the southern regions of Ukraine and in the Autonomous Republic of Crimea. In 311 points of the studied region, the ticks $\mathrm{D}$. reticulatus locations were found. According to the results of research, D. reticulatus was found in 79 points of Odessa, 45 - Mykolaiv, 44 - Kherson, 60 - Zaporizhia, 57 - Donetsk regions and in 26 points of the Autonomous Republic of Crimea. It was found that the locations of D. reticulatus ticks are located in the interval: the maximum northern point is 51044'54 " north. w. (Volyn region) and the southernmost point 45003 ' $05^{\prime}$ 'north. w. (Crimea). The westernmost point within Ukraine is located at 22042 ' 49 ' 'east. d. (Transcarpathian region) and the extreme eastern point - 38064 ' 80 ' 'east. w. (Donetsk region).

We have created an interactive map based on the medical geographic information system, which includes all the data obtained and above (Fig. 1).

The medical geoinformation system is created as specialized medical cartographic and analytical resource designed to collect, store, accumulate, analyze and display information about the spread of tick infections. The map provides analytical information on a wide range of tick infections in order to achieve a comprehensive view of the current state of human and animal morbidity and to develop an action plan to prevent and eliminate the negative impact.

The implementation of a geographic information system allows recording, storage and display of data on the distribution of tick-borne pathogens, as well as visualize information on the spread of tick infections in a user-friendly format (spreadsheets, charts and graphs with reference to the area).

Interactive maps show the online state of the of Ixodes ticks spreading, including D. reticulatus in a certain area (Fig. 2). It contains the most complete information on the frequency of occurrence of different stages of development of ticks (larvae, nymphs, adults) at a certain time, on certain nutrients, their epidemiological condition et al. These maps also contain information about the infection of people with various pathogens of infectious diseases transmitted by these ticks.

The use of an interactive map provides the collection of all this data in real time using a mobile cartographic application synchronized with the geographic information system. The cartographic application installed on the researcher's smartphone will allow to carry out systematic monitoring, accounting and control over the medical-geographical situation of the studied territory in real time. Using a system of queries and intelligent search of the electronic map, it is possible to perform a spatial analysis of the prevalence of diseases caused by ticks (babesiosis, anaplasmosis, piroplasmosis, etc.) and to monitor the real threats to public health [28].

\section{CONCLUSIONS}

Ticks of the species $D$. reticulatus are most often vectors of babesia, in particular Babesia canis, rickettsia-Rickettsia raoulti, anaplasma - Anaplasma phagocytophilum, Borrelia - Borelia burgdorferi s.l. and tick-borne encephalitis. D. reticulatus is most common in humid habitats near water bodies and in urbanized biocenoses, in which murine rodents are common, and which are often visited by domestic animals and humans. The main feeding hosts for genus $D$. reticulatus ticks are dogs, cows, horses, cats and humans. Only adult females and males feed on humans. Medical geographic information system allows to create electronic cartographic models for scientific research and practical use for systematic monitoring, accounting and control of the medical and geographical situation of the study area on the prevalence of ticks and morbidity.

\section{REFERENCES}

1. Bakken J.S. Human granulocytic ehrlichiosis. Clin. Infect. Dis. 2000; (31): 554-560.

2. Fedoniuk L. Ya. Morphological and physiological, biological and features of Acariana of the genus Ixodes (Latreille, 1795) - human ectoparasites in biogenocenoses of Ternopil region. Wiad. Lek.. 2019; 72(2): 224-229.

3. Mierzejewska E. J., Welc-Faleciak R., Karbowiak G. et al. Dominance Dermacentor reticulatus over Ixodes ricinus (Ixodidae) on livestock, companion animals and wild ruminants in eastern and central Poland. Experimental and Applied Acarology. 2015; 1(66): 83-101.

4. Dzięgiel B., Kubrak T., Adaszek Ł. et al. Prevalence of Babesia canis, Borrelia burgdorferi sensu lato, and Anaplasma phagocytophilum in hard ticks collected from meadows of Lubelskie Voivodship (eastern Poland). Bull Vet Inst Pulawy. 2014; 58:29-33.

5. Bonnet S., de la Fuente J., Nicollet P. et al. Prevalence of tick-borne pathogens in adult Dermacentor spp. ticks from nine collection sites in France. Vector Borne Zoonotic Dis. 2013;13:226-36.

6. Reye A.L., Stegniy V., Mishaeva N.P. et al. Prevalence of tick-borne pathogens in Ixodes ricinus and Dermacentor reticulates ticks from different geographical locations in Belarus. PLoS One. 2013;8:e54476).

7. Wójcik-Fatla A., Cisak E., Zając V. et al. Prevalence of tick-borne encephalitis virus in Ixodes ricinus and Dermacentor reticulates ticks collected from the Lublin region (eastern Poland). Ticks and Tick-borne Diseases. 2011; 2(1): 16-19.

8. Tomanovic S., Chochlakis D., Radulovic Z., Milutinovic M. Analysis of pathogen co-occurrence in host-seeking adult hard ticks from Serbia. Exp Appl Acarol. 2013; (59): 367-376.

9. Akimov A. Distribution of ticks of the genus Dermacentor (Acari, Ixodidae])in Ukraine. [A. Akimov, I. V. Nebogatkin].Vestnik zoologii. 2011; 45(1):35-40.

10. EmchukE.M. Fauna Ukrayini.lksodoviklischi. [Ukrainianfauna. Ixodes mites]. Kiyiv. Vidavnitstvo Akademiyi nauk USRR, 1960; (25)1: 168. (in Ukrainian).

11. Turyanin I. I. O svoeobraznyih ochagah iksodovyih kleschey v nekotoryih punktah Ukrainskih Karpat. [About some specific places of localization in Kapacian mountains of ixodes ricinus] V sb.: Ekologiya nasekomyih i drugih nazemnyih bespozvonochnyih Sovetskih Karpat. Uzhgorod: 1964, 85-87. (In Russian).

12. Akimov I. A., Nebogatkin I. V. Iksodovyie kleschi g. Kievaurbozoologicheskie iepizootologicheskie aspektyi [lxoeds ricinus in Kiev - urbozoological and epizootological aspects]. Vestnik zoologii. 2002; 36(1): 91-95. (In Russian).

13. Nebogatkin I.V. Ixodes ricinus ta Dermacentor reticulatus (Acari: Ixodida: Ixodidae) na pivdni Ukrayini [lxodes ricinus and Dermacentor reticulatus in the eastern Ukraine]. Ukrayinska entomofaunlstika. 2018; 9(1):43-57. (In Ukrainian). 
14. Kolonin G.V. Rasprostranenie iksodovyih kleschey Roda Dermacentor idr. [The spread of Ixodes Ricinus Dermacentor] G.V. Kolonin. M. Nauka. $1984,94 p$.

15. Uspenskaya I. G. Iksodovyie kleschi Moldavii i nekotoryie osobennosti ih ekologii. Avtoref. na soisk. uch. step. kand. biol. nauk. Kishenev: 1963,23p.

16. Byichkova E. I. Iksodovyie kleschi (Ixodidae) v usloviyah Belarusi: monografiya. Minsk: 2015,192p.

17. Biaduń W. New Habitats of Dermacentor reticulatus (Fabricius, 1794) in the Lublin Region. Pol. J. Environ. Stud. 2011;20(2): 63-266.

18. Paziewska A., Zwolińska L., Harris P. D. et al. The use of rodent species by larvae and nymphs of hard ticks (Ixodidae) in two habitats in northeastern Poland.Experimental and Applied Acarology. 2010; 50: 79.

19. Zygner W.1., Górski P., Wedrychowicz H. New localities of Dermacentor reticulatus tick [vector of Babesia canis canis] in central and eastern Poland. Pol J Vet Sci. 2009;12(4): 549-55.

20. Chitimia-Dobler L. Spatial distribution of Dermacentor reticulatus in Romania.Veterinary Parasitology. 2015;214 (1-2): 219-223.

21. Bullová E., Lukáň M., Stanko M., Pet'ko B. Spatial distribution of Dermacentor reticulatus tick in Slovakia in the beginning of the 21st century. Veterinary Parasitology. 2009; 165 (3-4): 357-360.

22. Földvári G., Šroký P., Szekeres S. et al. Dermacentor reticulatus: a vector on the rise. Parasit Vectors. 2016; 9(1): 314.

23. GáborFöldvári R. Ixodid tick species attaching to dogs in Hungary. Veterinary Parasitology. 2005; 129 (1-2):125-131.

24. Fedonyuk L.Ya., Podobivskyi S. S., Korda M. M. et al. Morfo-fiziologichni osoblivosti ta medichne znachennya Iksodovih klischiv rodiv Ixodes Latr. ta Dermacentor Fabr.- ektoparazitiv lyudini I tvarin u Zahidniy Ukraiini [Morpho-physiological features and medical significance of ixodic mites of Ixodes latr. and Dermacentor fabr. generations - humans and animals ectoparasites in western Ukraine. Svit meditsini ta biologiyi. 2018; 1(63):173-177. (In Ukrainian).

25. Podobivkyi S. S. Comparative characteristic of the morpho-physiological parameters, biology and epidemiology of Ixodidae (Ixodes and Dermacentor). DeutscherWissenschafsherold. Gerrman Science Herald. 2018(2): 10-12.

26. Podobivskiy S. S., Fedonyuk L. Ya., Korda M. M. et al. Vprovadzhennya medichnoyi geoinformatsiynoyi sistemi pri doslidzhennyah iksodovih klischlv i klischovih infektsiy v Ukrayini. Infektslynl hvorobi. 2019; 3(97): 38- 45. (In Ukrainian).
The research was performed as part of the initiative research work "Study of species diversity, distribution, biology and ecology of Ixodes ticks in the western region of Ukraine", the state registration number: 0117 U003025.

\section{ORCID and contributionship:}

Larysa Y. Fedoniuk: 0000-0003-4910-6888 A, D, E, F

Stepan S. Podobivskiy: 0000-0002-6667-1478 B, C

Iryna B. Pryvrotska: 0000-0002-4610-4943 ${ }^{D}$

Olga M. Marchuk: 0000-0002-9089-0873 ${ }^{B}$

Olena A. Miklashevska: 0000-0002-3938-7893 C, F

\section{Conflict of interest:}

The Authors declare no conflict of interest.

\section{CORRESPONDING AUTHOR Stepan S. Podobivskiy \\ Horbachevsky Ternopil National Medical University \\ Ministry of Health of Ukraine \\ 1 Maidan Voli st., 46001 Ternopil, Ukraine \\ e-mail: podobivskiy@tdmu.edu.ua}

Received: 11.04 .2021

Accepted: 29.07.2021

A - Work concept and design, B - Data collection and analysis, C - Responsibility for statistical analysis, D-Writing the article, $\mathbf{E}$-Critical review, $\mathbf{F}$ - Final approval of the article 